\title{
In-Depth Observation of Video Gamers
}

\author{
Jorge Alves Lino and Ben Salem \\ Department of Industrial Design, Eindhoven University of Technology, The Netherlands \\ jorge@jorgelino.com, mail@bsalem.com
}

\begin{abstract}
In this poster we present the observations results of video gamers playing Halo 3 on the X-Box console. We report on the data we have gathered thanks to a Noldus Observer system. These data relate to gamer behaviour, game actions and basic biofeedback.
\end{abstract}

Keywords: Game, gamer observation, Behaviour, physiological data.

\section{Introduction}

Current research demonstrates that the video game industry has been increasing its presence in the field of Entertainment, more and more overcoming the supremacy that before the Film Industry had, this is for example very clear in the US [1]. The audience of video games is becoming more diverse: gaming is no longer an exclusive entertainment activity by male young adults, while elderly and women are becoming more proactive as video game users and players [2]. Video games are becoming a major part of our entertainment habits. However we feel little has been investigated on the way people actually play video games. It is crucial to extend and refine the observation of video gamers, up to a level where we can draw correlations between gamer behaviour, character actions, and physiological parameters. We are also interested in assessing differences if any between genders, socio-cultural and age groups.

\section{Method}

We propose to conduct video game sessions, where players should go through a stage of the game. During this playing experience, we will observe simultaneously the gamer behaviour, character actions as directed by the gamer and physiological parameters, and compare the different correlations between these factors. We believe that the observation of galvanic skin conductance and heart rate can provide us an overview of the state of arousal of the player, which combined with the behaviour adopted, can provide us valuable results on dexterity and experience of the gamer. During this process, the fact that we are conducting observations with both male and female subjects will allow us to conclude whether there are gender differences or not.

\section{Installation}

For these experiments, we used the GameLAB facility of the Department of Industrial Design of the Eindhoven University of Technology. 
The installation set for the gaming observations includes the following equipment:

Hardware: 42-inch LCD Display, Microsoft X-Box, sound system with 4 speakers and 1 sub-woofer, the Biopac, 3 observing cameras.

Software: Noldus Observer, Noldus Acknowledge, Microsoft Halo 3.

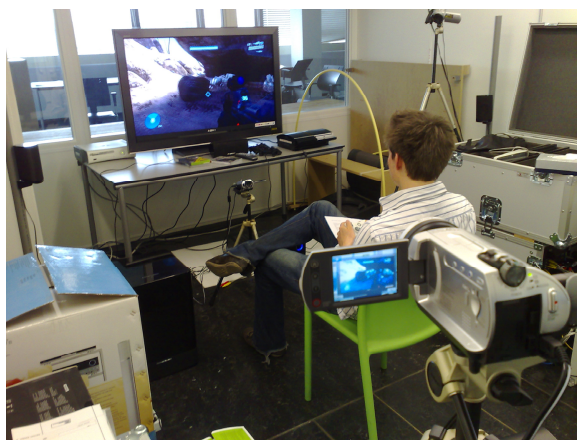

Fig. 1. Our GameLAB installation

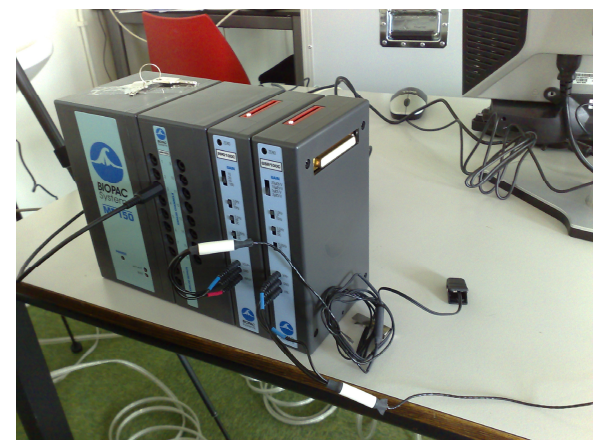

Fig. 2. The Biopac Module used for biofeedback observations

\section{Observations}

Subjects between 20 and 30 both male and female are asked by the test moderator to participate in the survey. On the scheduled appointment, they are individually taken to the GameLAB, where a previously prepared A4 sheet with the game instructions is provided, and they are installed in the facilities and connected to the observation system. Subjects carefully read the instructions in order to have an overview on the purpose of the task within the game, and they are asked to play a 2 minute trial to experience the different instructions. Before starting the moderator fixes sensors on the fingers and ear of the test subjects. While this trial period is going on, the test moderator checks if the observation system is well connected to the subject, and working properly.

Once basic understanding of the task is achieved, and the observation system is checked, subjects initiate a campaign, where they perform as real players. While playing, the following parameters are being observed:

- Physical behaviour (expression, manipulation of remote, body language)

- Character behaviour (the kind of strategy is adopted within the game)

- Biofeedback during gaming experience (Heart rate, galvanic skin conductance)

Using the observation software Noldus Observer allowed us to observe simultaneously biofeedback, namely subjects' heart rate and galvanic skin conductance, and the gaming experience itself. Our lab is also equipped with 3 cameras, with different viewpoints of the player. There was an overall camera view of the whole installation with the player in the middle. There was also a camera pointing mainly at the gamer face to record facial expression variations. Finally a third camera was pointing at the player body to identify body language and game controller 
manipulations and actions. The software allows a complete synchronisation of the different channels of observation. The different observations can therefore be overlapped in order to detect patterns of activities and highlight correlations between the different parameters we were observing.

\section{Results}

Regarding the game actions, we observed that male subjects have a direct approach to dangerous situations within the game, by going straight forward to the action point of the game. Female players adopt a defensive and self-protective strategy of their character, by approaching the action point of the game with prudence. Players were inexperienced with this game, but didn't have difficulty in finishing the task they were required.

Regarding the physiological observations and the player's behaviour, the process is still ongoing and the results are still partial, depending on further observations in order to sustain conclusive observations.

\section{Discussion}

So far, we have observed a clear difference between the male and female players, regarding the way they conduct their character actions. Male subjects engage frontally in the conflict within the game, whereas female subjects assume a strategic approach. Given the fact that the gamers were inexperienced and they showed no substantial difficulties in accomplishing the task, we concluded that this game has a good quality of design of interaction. Given the gender differences in the observations, we believe it would be interesting if such interaction design was adaptive to the gender of the players. This could be achieved, for example, by creating both male and female characters within the game, in which the role the gamer plays in the game would be related with their own gender (and the behaviour particularities that it involves).

\section{References}

1. Davis, J.P., Steury, K., Pagulayan, R.: A survey method for assessing perceptions of a game: The consumer playtest in game design. International Journal of Computer Game Research 5(1) (2005)

2. 2008 Essential facts about the computer and video game industry. Entertainment Software Association, Washington, DC, USA (2008) 